\title{
First Regulatory Qualification of a Novel Digital Endpoint in Duchenne Muscular Dystrophy: A Multi-Stakeholder Perspective on the Impact for Patients and for Drug Development in Neuromuscular Diseases
}

\author{
Laurent Servais ${ }^{\mathrm{a}, \mathrm{b}}$ Eric Camino ${ }^{c}$ Aude Clement $^{\mathrm{d}}$ Craig M. McDonald $^{\mathrm{e}}$ Jacek Lukawy ${ }^{\mathrm{d}}$ \\ Linda P. Lowes $^{f}$ Damien Eggenspielerg Francesca Cerreta ${ }^{\mathrm{h}}$ Paul Strijbos $^{\mathrm{d}}$ \\ aMDUK Oxford Neuromuscular Centre, Department of Paediatrics, University of Oxford, Oxford, UK; ${ }^{b}$ Division \\ of Child Neurology, Centre de Référence des Maladies Neuromusculaires, Department of Pediatrics, University \\ Hospital Liège and University of Liège, Liege, Belgium; 'Parent Project Muscular Dystrophy, Hackensack, NJ, USA; \\ ${ }^{d}$ F. Hoffmann-La Roche Ltd, Basel, Switzerland; eUniversity of California Davis Health, Sacramento, CA, USA; ${ }^{f} A b i g a i l$ \\ Wexner Research Institute at Nationwide Children's Hospital, The Ohio State University, Columbus, OH, USA; \\ gSYSNAV, Paris, France; hEuropean Medicines Agency, Amsterdam, The Netherlands
}

\section{Key Messages}

- European Union regulators consider SV95C an acceptable secondary efficacy endpoint in clinical trials of ambulant DMD patients $\geq 5$ years of age for regulatory purposes.

- Importantly, regulators stipulate that SV95C should be measured by using a validated and suitable wearable device that can quantify ambulation directly and continuously in a real-world environment. The only device currently validated for the digital assessment of SV95C in clinical trials is ActiMyo ${ }^{\circledR}$, a class I medical device that includes two strap-based sensors worn on the ankle.

- Recent regulatory qualification of SV95C constitutes a major breakthrough in the adoption of novel digital endpoints in drug development. Wearable technology allows functional assessments to be transferred to the community environment - reducing patient and caregiver burden in clinical trials. By providing high-quality and meaningful efficacy data, they will enable more efficient drug development and could expedite registration and access of new therapies for diseases with unmet medical needs.

\section{Keywords}

Duchenne muscular dystrophy · Digital endpoints .

Neuromuscular disease

\section{Abstract \\ Background: Functional outcome measures used to assess efficacy in clinical trials of investigational treatments for rare neuromuscular diseases like Duchenne muscular dystrophy}

(DMD) are performance-based tasks completed by the patient during hospital visits. These are prone to bias and may not reflect motor abilities in real-world settings. Digital tools, such as wearable devices and other remote sensors, provide the opportunity for continuous, objective, and sensitive

Disclaimer: The views expressed in this article are the personal views of the author(s) and may not be understood or quoted as being made on behalf of or reflecting the position of the regulatory agency/agencies or organizations with which the author(s) is/are employed/affiliated.
(C) 2021 The Author(s).

Published by S. Karger AG, Basel

This is an Open Access article licensed under the Creative Common Attribution-NonCommercial-4.0 International License (CC BY-NC) (http://www.karger.com/Services/OpenAccessLicense), applicable to the online version of the article only. Usage and distribution for commercial purposes requires written permission.
Correspondence to:

Paul Strijbos, paul.strijbos@ roche.com 
measurements of functional ability during daily life. Maintaining ambulation is of key importance to individuals with DMD. Stride velocity 95th centile (SV95C) is the first wearable acquired digital endpoint to receive qualification from the European Medicines Agency (EMA) to quantify the ambulation ability of ambulant DMD patients aged $\geq 5$ years in drug therapeutic studies; it is also currently under review for the US Food and Drug Administration (FDA) qualification. Summary: Focusing on SV95C as a key example, we describe perspectives of multiple stakeholders on the promise of novel digital endpoints in neuromuscular disease drug development.

(C) 2021 The Author(s) Published by S. Karger AG, Basel

\section{Introduction}

Duchenne muscular dystrophy (DMD) is an X-linked recessive disorder caused by mutations in the gene that encodes the protein dystrophin [1]. DMD is characterized by muscle degeneration that worsens with age. Despite the availability of glucocorticoid treatment, patients are expected to be nonambulatory and wheelchair dependent by age 13 [2,3]. A number of different therapeutic interventions have been studied in DMD, including dystrophin gene therapy [4]. Despite recent US Food and Drug Administration (FDA) approvals, unmet needs for patients with DMD remain [4], as none of the currently available treatments stop the progressive course of the disease.

Functional outcome measures for assessing patients with neuromuscular disease in clinical trials have traditionally consisted of timed tests and motor scales assessed during hospital visits $[5,6]$. One common complaint about traditional measures is that they may not capture real-world benefits [7]. Moreover, timed tests may be burdensome to patients with more severe disease. The most frequently used clinical outcome assessments (COA) in clinical trials, the 6-min walk test [2] and the North Star Ambulatory Assessment [8], assess mobility in ambulatory patients and are designed to evaluate peak patient performance intermittently within a controlled environment (i.e., on assigned days within a hospital or clinic). Such assessments may be prone to bias and do not always accurately represent function during daily life [5, 9]. Multiple external factors including motivation, fatigue, time of day, maturity, developmental stage, and behavior are known to affect performance on these assessments $[10,11]$. These issues can be compounded in younger children or those with attention or learning difficulties $[12,13]$. Together, these challenges may serve as barriers to successful drug development in DMD [14].

In January 2017, Duchenne UK, Parent Project Muscular Dystrophy (USA), Duchenne Parent Project (Netherlands), and Duchenne Parent Project Onlus (Italy) cofunded a workshop to review endpoints currently used in DMD clinical trials and to assess gaps [5]. The group indicated that advances in digital tools such as wearable devices and other remote sensors provide the opportunity to develop clinically relevant outcome measures through the collection of reliable, objective efficacy data from the patient's daily life. Indeed, the adoption of qualified digitally measured endpoints provides an opportunity to accurately and continuously assess the potential benefits of an investigational therapy in a real-world setting [15].

Maintaining ambulation is of major importance to individuals with DMD. Hence, the ability to measure this parameter accurately and objectively in a community setting is critical for the assessment of investigational therapies. Digital-based wearable sensors - including biaxial
Fig. 1. What is SV95C? Description of SV95C and the data used to support its qualification by the European Medicines Agency. SV95C, stride velocity 95th centile.

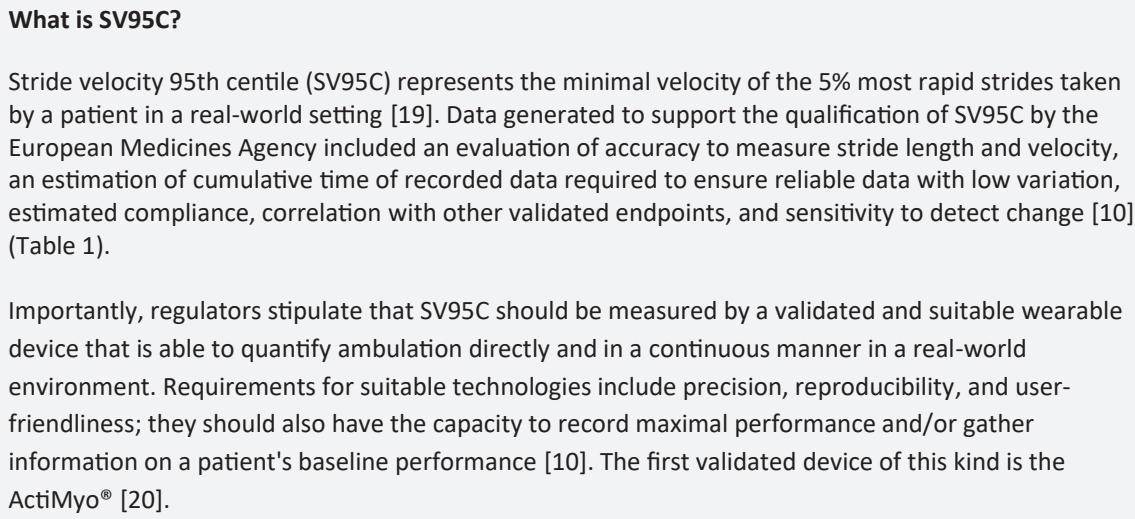


Fig. 2. What is ActiMyo ${ }^{\circledR}$ ? Description of the ActiMyo ${ }^{\circledR}$ device and its validation for gathering SV95C data [20-22]. SV95C, stride velocity 95 th centile.

\section{What is ActiMyo ${ }^{\oplus}$ ?}

ActiMyo ${ }^{\circledR}$ is a Class I medical device composed of a base station for charging and data transferral, and 2 strap-based wearable sensors. ActiMyo ${ }^{\circledR}$ uses precision magneto-inertial sensors that continuously measure acceleration, velocity and angular movements over prolonged periods with high accuracy in the home environment. The device was designed and validated with patient input and thus has the potential to define meaningful improvements and clinical trial outcome measures for individual patients [20]. Based on EMA-defined precision requirements, the wearable sensor ActiMyo ${ }^{\circledR}$ is currently the only suitable and validated device for gathering SV95C data for use in clinical trials. Other ActiMyo ${ }^{\circledR}$ acquired endpoints are likely to be developed and validated in both ambulatory and non-ambulatory neuromuscular diseases $[21,22]$. accelerometers - have been studied in DMD; DMD patients differ from typically developing youth in total steps and step frequency at the highest step rates or speeds [16]. The digital parameters of total steps per day and steps at the highest rate decline with disease progression and are correlated with traditional endpoints, such as 10 -m velocity in meters per second $[17,18]$. However, these monitors only provide accurate step counts and do not measure the instantaneous stride length of each stride. In addition, the definitions of high step rate have been arbitrarily set and are not based on a total distribution of stride velocities. A recent long-term natural history study of laboratory-based gait kinematic parameters in DMD demonstrated that stride length declines more than cadence (steps per minute) with disease progression - as does self-selected walking speed (the product of step length and cadence) [18]. Furthermore, steroids produce an increase in stride length in the 1-year period typically employed in clinical trials, which independently confirms the need to precisely measure step length and stride velocity in DMD [17].

Stride velocity 95th centile (SV95C), measured at the ankle by using a valid and suitable wearable device, is the first digital endpoint to receive qualification from the European Medicines Agency (EMA) (Fig. 1). SV95C quantifies a patient's ambulation ability directly and reliably in a continuous manner in the home environment as an indicator of maximal performance. EU regulators consider it an acceptable secondary endpoint in clinical trials of ambulant DMD patients $\geq 5$ years of age as well as a qualified measure to quantify a patient's baseline performance in such studies (EMA 2019). SV95C is also currently under review for FDA qualification (FDA 2018). ActiMyo ${ }^{\circledR}$, a class I medical device (Fig. 2) that includes two strapbased sensors worn on the ankles and/or wrists, was used to collect SV95C data for the endpoint qualification.

In this paper, we explore the perspectives of multiple stakeholders on the use of digital measures and the asso-

A Multi-Stakeholder Perspective on a

Novel Digital Endpoint in DMD ciated digital tools in clinical trials of DMD. We discuss the potential impact of novel digital endpoints, like SV95C, on the DMD drug development paradigm.

\section{The Patient and Caregiver's Perspective}

Clinical outcome measures that reliably evaluate the wide range of DMD patient experiences, which vary significantly from childhood through adulthood, are critically needed. In addition, currently used functional outcome assessments are not able to detect change across the full spectrum of disease severity. For example, if the 6-min walk test or the North Star Ambulatory Assessment is the primary endpoint for a study, those who score poorly at baseline may be excluded from clinical trials on the basis that they are expected to become nonambulant over the ensuing 12 months $[6,23]$. This pitfall impacts trial demographics, as older patients and those who are nonambulant can be excluded from clinical trials.

There are a number of fundamental challenges for patients with DMD who wish to participate in trials. As the duration of clinical trials continues to increase, the cost and burden associated with participation also increase [24]. In addition, patients and caregivers must find means of transportation to sometimes distant study sites, which can involve time missed from work or school as well as upfront travel costs [25]. These issues again limit who can participate in clinical trials, as the most severely impacted patients are typically not eligible to participate.

Moreover, visits to hospitals may be interrupted by crises, such as the COVID-19 pandemic, which has disrupted several clinical trials in which the primary endpoint was a hospital-based COA. Decentralization, through the use of endpoints measured by wearable technology in a home and community environment, may provide the opportunity for more patients to participate, as there would be less reliance on burdensome functional assessments and, crucially, trials could be designed with the patient in mind. 


\section{The Clinician's Perspective}

Clinicians are acutely aware of the need for outcome measures for DMD and other neuromuscular diseases that can accurately assess "typical daily function" across a diverse spectrum of patients. Standardization of currently used assessments and assessor training across study sites in a clinical trial setting can be challenging because outcomes rely on subjective human observation and can vary based on disease stage and baseline level of function [26]. Furthermore, traditional assessments are intermittent, providing only a snapshot of functional ability. For example, children in the early stages of DMD have a capacity reserve that may hamper objective assessment at a single point in time if incentivized [10, 11]. In clinical practice, standardization can be even more challenging due to the variation inherent to a real-world setting.

Digital tools that allow measurements of functional outcomes in an unprecedented longitudinal manner, using continuous real-world data collection, could provide valuable insight into disease progression. Once they are well characterized across the spectrum of disease, digital measures could be used to inform optimal patient management or aid in differential diagnosis. Appropriate endpoints derived from wearable devices may also decrease the time required to perform assessments while adding important real-world data to build confidence in putative therapies. The availability of objective and comparable data to complement a clinician's subjective assessment could provide support for decisions to continue, stop, or change a particular therapy. Moreover, gathering precise and continuous measures could assist with tailoring a treatment plan to a particular patient's response, such as with steroid treatment, for which efficacy can be evaluated in a short period of time [27].

While wearable-derived clinical outcome measures have important implications in the clinical development of new therapies, there are currently barriers to their widespread use in clinical practice. For one, there are distinctions between what constitutes an ideal digital endpoint for clinical trials as opposed to a clinical practice tool. The most important factors for clinical trials are ease of use of the wearable device for the patients and the ability to acquire robust and comprehensive efficacy data, while the most important consideration for clinical practice is how user-friendly the assessment tool is for physicians. Digital drug development tools - such as ActiMyo ${ }^{\circledR}$ - are currently not designed to fit into the clinical workflow, as they would require physicians to access the digital output. The digital solution would then need to be registered with the corresponding medical intended use and be accepted as standard of care and readily available to provide decision support to physicians in clinical practice.

\section{The Technology Company's Perspective}

Technology companies are important partners in the development of new therapies for DMD. However, digital endpoint technology development is more nuanced than just the creation of a wearable device or supplying off-theshelf hardware. Expertise in the development of both precision hardware and algorithms, combined with effective data management, is essential.

Early collaboration between technology companies and all other stakeholders is crucial to allow for the creation of devices that measure meaningful endpoints. For example, working closely with patient organizations will lead to the development of "fit-for-purpose" devices that are precise enough to provide accurate and reliable measurements while also being user-friendly for patients. Effective management of data security is also vital to protect the privacy of patients. Companies should partner with physicians to validate the level of performance of devices, define meaningful measures, and build capabilities to integrate devices into clinical studies; this could include training and support for clinicians, compliance monitoring, and data analysis. Engagement with industry sponsors is also important to build normative databases for healthy controls, as well as untreated and treated patients. Finally, at all stages of the development process, technology companies should cooperate with regulatory agencies to qualify derived endpoints.

Regarding intellectual property rights, endpoints are currently public domain and should remain solution agnostic, provided a validated and suitable solution is used. The solution/devices are proprietary. While strategies vary by company, companies might protect the solution/ device through a combination of expertise, mastery in execution, intellectual property, and trade secrets.

\section{The Drug Developer's Perspective}

The adoption of patient-facing digital technology in clinical trials has the potential to improve the patient experience, which may lead to increased participation, retention, and compliance [28]. In DMD drug development programs, the use of digital endpoints derived from wearable technology that are easy to use at home and have been qualified to reflect clinically meaningful functional change in a real-world environment can reduce the burden of clinical trial participation and increase patient engagement [10]. Such endpoints may enable studies to be more inclusive by widening the eligibility criteria and 
Table 1. Data summaries from validation studies used to support SV95C digital endpoint qualification [10]

\begin{tabular}{|c|c|c|}
\hline Objective & Method & Results \\
\hline $\begin{array}{l}\text { Demonstrate that the foot trajectory of } \\
\text { ambulant patients as assessed by the } \\
\text { magneto-inertial sensor corresponds to the } \\
\text { real distance measured manually }\end{array}$ & $\begin{array}{l}\text { Simultaneously measured distance during the } \\
6 \text {-min walk test using the wearable device/ } \\
\text { system and the classical method for } 31 \text { tests } \\
\text { performed by } 23 \text { patients in a broad range of } \\
\text { clinical conditions }\end{array}$ & $\begin{array}{l}\text { The difference between the distance measured during } \\
\text { the } 6 \text {-min walk test using the wearable device/system, } \\
\text { and the corrected reference was within } 5 \%\end{array}$ \\
\hline $\begin{array}{l}\text { Assess the reliability of proposed gait } \\
\text { variables measured by the wearable device } \\
\text { and system }\end{array}$ & $\begin{array}{l}\text { Studied the relationship between the recording } \\
\text { period averaging and the variability of the } \\
\text { measure by tracing the SYSNAV variance in } \\
28 \text { patients tested in a noncontrolled setting }\end{array}$ & $\begin{array}{l}\text { Good stability with low variability from } 2.22 \text { up to } \\
4.41 \% \text { for the } 95 \text { th percentile of stride length and } 95 \text { th } \\
\text { percentile of stride velocity, respectively, for } 180 \text { h of } \\
\text { wearable device and system use }\end{array}$ \\
\hline $\begin{array}{l}\text { Cross-validate these measures with } \\
\text { reference to clinical outcomes }\end{array}$ & $\begin{array}{l}\text { Studied the relationship between stride } \\
\text { velocity, stride length, and distance per hour } \\
\text { and the 6-min walk test, North Star } \\
\text { Ambulatory Assessment score, and 4-stair } \\
\text { climbing test score in } 45 \text { DMD patients }\end{array}$ & $\begin{array}{l}\text { Good correlation of } 95 \text { th percentile of stride length } \\
\text { and } 95 \text { th percentile of stride velocity with the baseline } \\
6 \text {-min walk test }(0.68 \text { and } 0.54 \text {, respectively) and with } \\
\text { the North Star Ambulatory Assessment ( } 0.78 \text { and } \\
0.64 \text {, respectively) }\end{array}$ \\
\hline $\begin{array}{l}\text { At } 6 \text { months, study the sensitivity to change } \\
\text { of variables measured by the wearable } \\
\text { device and system }\end{array}$ & $\begin{array}{l}\text { Studied } 31 \text { patients for a full } 6 \text {-month period. } \\
\text { Studied a subset of } 20 \text { patients (who were older } \\
\text { than } 6 \text { years and walking }<450 \mathrm{~m} \text { in the } 6 \text {-min } \\
\text { walk test) for over } 6 \text { months. Within this } 20 \\
\text { patient subset, studied } 7 \text { patients for over } 1 \\
\text { year }\end{array}$ & $\begin{array}{l}\text { Significant decline in } 95 \text { th percentile of stride length } \\
(2.4 \%) \text {, median stride velocity }(4.7 \%) \text {, and } 95 \text { th } \\
\text { percentile of velocity }(8.5 \%) \text { over } 6 \text { months for } \\
\text { patients older than } 6 \text { with a baseline } 6 \text {-min walk } \\
\text { distance below } 450 \mathrm{~m}\end{array}$ \\
\hline
\end{tabular}

DMD, Duchenne muscular dystrophy; SV95C, stride velocity 95th centile.

thereby allowing for the assessment of investigational therapies in a broader population.

The efficient generation of accurate and reliable efficacy data for an investigational treatment is essential to the drug development process [6]. Validated digital endpoints measured via wearable devices allow for the continuous collection of data relevant to patients' daily lives. By enabling improved patient insight and high data integrity, it is likely these endpoints will help expedite patient access to effective therapies.

DMD is a slowly progressive disease that worsens over time [29]. For this reason, registration trials in DMD can be long and require many patients to evaluate efficacy. This challenge is common for trial recruitment in rare disease [30]. Because digital measures such as SV95C allow deep phenotyping of disease characteristics in each individual patient, they may more sensitively assess changes in disease progression, thus reducing the sample size or duration required for a clinical trial when selected as primary endpoints. Following approval of an effective therapy, digital measures may allow for the collection of individual efficacy data or offer personalized disease monitoring.

Implementation of novel digital endpoints into clinical trials should be supported with training and education of the patient, carer, and support staff to maximize user acceptance and to minimize missing data [10]. Further- more, the principles of Good Clinical Practice, data privacy, and protection should be respected [15].

\section{The Regulator's Perspective}

Stakeholders, including technology companies, patient groups, and consortia working to develop digital endpoints for use in drug development, are encouraged to engage regulators early in the development process. Both the FDA and EMA have regulatory pathways that allow collaboration throughout the development and validation of a novel COA. The FDA Drug Development Tools COA Qualification Program [31] and the EMA qualification procedure of novel methodologies for medicine development [32] are available to facilitate regulatory endorsement of novel digital endpoints. However, regulatory qualification of a COA by the FDA or EMA is not a prerequisite for use as an endpoint in drug therapeutic trials.

SV95C, measured by using a valid and suitable wearable device in ambulant patients aged $\geq 5$ years with DMD, was the first digital COA to secure an EMA qualification opinion as a secondary endpoint in pivotal or exploratory clinical trials (Fig. 1). Table 1 provides a summary of the validation studies that supported qualification of the digital endpoint.

This particular qualification provided valuable learnings on the innovative elements to be considered for re- 
viewing such digital endpoints, including the data requirements. These insights were also leveraged in a new EMA Q\&A on the qualification of digital technologybased methodologies to support approval of medicinal products [33].

Regarding use as the primary endpoint for pivotal trials in this setting, EU regulators consider that more robust data on the long-term correlation of SV95C with currently used tests (including increased sample size, longer follow-up, and expanded normative data) are needed [19]. Anchor-based approaches (e.g., relating a drop in SV95C with loss of ambulation) would help elucidate the clinical relevance of changes in SV95C. Additionally, the system currently does not offer data about walking, falls, stairs, time to stand, and correlation with patient wellbeing, and further assessment in younger and nonambulant patients is of interest.

The clinical relevance of the proposed minimal clinically important difference (MCID) should also be further justified. It would be particularly informative for anchorbased approaches that estimate MCID for SV95C to relate a drop in the SV95C to function in daily life or to another qualified clinician or patient assessment [19]. Previous analyses have indicated consistency between the MCID for SV95C and those for the 6-min walk test and the North Star Ambulatory Assessment [10, 19]. Corresponding sample size estimates for SV95C illustrate the significant impact of the use of this digital variable as the primary endpoint in pivotal trials in DMD: it has been estimated that approximately 30 patients per arm would be required to detect the stabilization of the disease by the investigational medicinal product over a 6 -month period using SV95C. This contrasts with the group size of 100 or more that is required to demonstrate stabilization using the 6-min walk test or North Star Ambulatory Assessment as the primary endpoint [10]. This example highlights the tremendous potential that highly validated and qualified digital endpoints have, both in terms of reducing patient burden of trial participation and study design.

\section{Conclusions and Future Directions}

The recent regulatory qualification of a digital measure of ambulation, SV95C, has established a path for the adoption of wearable technology in DMD registration trials and is considered a crucial precedent by multiple stakeholders for the development of novel digital endpoints in neuromuscular diseases. This qualification may advance the assessment of clinically meaningful func- tional improvements in DMD, as well as other neuromuscular diseases, by providing reliable and valid measures of functioning in a real-world setting.

Indeed, the FDA/Foundation for the National Institutes of Health has expressed considerable interest in this endpoint, which is also being processed through the FDA COA Qualification Program [31, 34]. The FDA qualification process consists of three steps: a letter of intent, a qualification plan, and a full qualification package. As of this writing, the letter of intent has been accepted, and submission of the qualification plan is being planned.

Wearable technology enables the transfer of functional assessments to the community environment, providing clinically meaningful outcomes for patients and caregivers and significantly reducing the burden associated with participation in clinical trials [35]. Digital endpoints will also likely make the development and registration of new therapies possible for a broader group of patients, expanding patient access and generating data to better inform healthcare decisions $[35,36]$.

Validation and regulatory acceptance are critical for the adoption of new digital endpoints [37]. Clinical meaningfulness, content validity, relationship with natural disease history, sensitivity to therapeutic intervention, relationship with current gold standard assessments, and community support must be established. Further, methods for the analysis of data derived for each endpoint, including strategies for handling intercurrent events (missing data), need to be defined.

A critical success factor for novel digital endpoint development is early multi-stakeholder engagement in a precompetitive environment, including the sharing of technology and the creation of confidential collaborative data platforms. Simultaneously, refinement and alignment of the regulatory procedures for qualification by the EMA and FDA is needed to facilitate timely validation and utilization of digital endpoints in drug therapeutic studies.

This study outlines strategies for the successful adoption of clinically meaningful digital measures in drug development from multi-stakeholder perspectives. SV95C provides the first example of a qualified digital endpoint and paves the way for transformation in the DMD therapeutic landscape.

\section{Acknowledgments}

The authors thank MediTech Media for providing medical writing support, which was funded by F. Hoffmann-La Roche Ltd., Basel, Switzerland, in accordance with Good Publication Practice (GPP3) guidelines (http://www.ismpp.org/gpp3). 


\section{Conflict of Interest Statement}

Dr. Servais reports grants and personal fees from Roche, grants and personal fees from AveXis, grants and personal fees from Biogen, personal fees from Cytokinetics, grants and personal fees from Dynacure, personal fees from Audentes, personal fees from Santhera, personal fees from Sarepta, personal fees from Affinia, personal fees from Pfizer, personal fees from REGENXBIO, personal fees from Lupin Pharmaceuticals, and personal fees from FibroGen, outside the submitted work; in addition, Dr. Servais is a coinventor of a device for measuring upper limb activity: "method for estimating the physical activity of an upper limb" and a coinventor on a patent for a method to estimate power at the upper limb level (this method can be used by ActiMyo ${ }^{\circledR}$ ). Dr. Servais has no financial return from these patents, which are held by his previous institution. Dr. Clement, Dr. Lukawy, and Dr. Strijbos are or were employees of F. Hoffmann-La Roche and have nothing else to disclose. Dr. McDonald reports grants from Capricor, grants from Catabasis, grants from Edgewise Therapeutics, grants from Epirium Bio, grants from Italfarmaco, grants from Pfizer, grants from PTC Therapeutics, grants from Santhera Pharmaceuticals, grants from Sarepta Therapeutics, other from Capricor, other from Catabasis, other from PTC Therapeutics, other from Santhera Pharmaceuticals, and other from Sarepta Therapeutics, outside the submitted work. Dr. Eggenspieler has nothing to disclose other than his affiliation with SYSNAV, a company that collaborated with the Institute of Myology to create ActiMyo ${ }^{\circledR}$. Dr. Eggenspieler is an employee of SYSNAV. Dr. Cerreta reports working for the European Medicines Agency. She disclaims that these opinions are presented in her personal capacity and do not represent the view of the Agency. Dr. Lowes and Dr. Camino have nothing to disclose.

\section{Funding Sources}

F. Hoffmann-La Roche funded the development of this review.

\section{Author Contributions}

L.S. participated in the design of the manuscript, drafted part of the content, critically reviewed the drafts, and approved the final version. D.E. participated in the discussions with the group of authors to shape the different perspectives and more specifically drafted part of "The Technology Company's Perspective" section. J.L. participated in the design of the manuscript, drafted part of the content, critically reviewed the drafts, and approved the final version. E.C. participated in the design of the manuscript, critically reviewed the drafts, and approved the final version. F.C. critically reviewed the drafts and approved the final version. L.P.L. critically reviewed the drafts and approved the final version. A.C. participated in the design of the manuscript, drafted part of the content (more specifically "The Drug Developer's Perspective" and "The Regulator's Perspective" sections), reviewed the drafts, and approved the final version. C.M.M. helped draft part of the content, critically reviewed the drafts, and approved the final version. P.S. participated in the design of the manuscript, drafted part of the content, critically reviewed the drafts, and approved the final version.

\section{References}

1 Falzarano MS, Scotton C, Passarelli C, Ferlini A. Duchenne muscular dystrophy: from diagnosis to therapy. Molecules. 2015 Oct;20(10): 18168-84.

2 McDonald CM, Henricson EK, Han JJ, Abresch RT, Nicorici A, Atkinson L, et al. The 6-minute walk test in Duchenne/Becker muscular dystrophy: longitudinal observations. Muscle Nerve. 2010 Dec;42(6):966-74.

3 Ryder S, Leadley RM, Armstrong N, Westwood M, de Kock S, Butt T, et al. The burden, epidemiology, costs and treatment for Duchenne muscular dystrophy: an evidence review. Orphanet J Rare Dis. 2017 Apr;12(1):79.

4 Verhaart IEC, Aartsma-Rus A. Therapeutic developments for Duchenne muscular dystrophy. Nat Rev Neurol. 2019 Jul;15(7):37386.

5 Straub V, Mercuri E; DMD Outcome Measure Study Group. Report on the workshop: meaningful outcome measures for Duchenne muscular dystrophy, London, UK, 30-31 January 2017. Neuromuscul Disord. 2018 Aug; 28(8):690-701.

6 Montes J, Kaufmann P. Outcome measures in neuromuscular diseases. In: Darras B, Jones Jr HR, Ryan M, De Vivo D, editors. Neuromus- cular disorders of infancy, childhood, and adolescence. 2nd ed. San Diego: Academic Press; 2015. p. 1078-89.

7 Ganea R, Jeannet PY, Paraschiv-Ionescu A, Goemans NM, Piot C, Van den Hauwe M, et al. Gait assessment in children with Duchenne muscular dystrophy during long-distance walking. J Child Neurol. 2012 Jan;27(1): 30-8.

8 Ricotti V, Ridout DA, Pane M, Main M, Mayhew A, Mercuri E, et al. The NorthStar Ambulatory Assessment in Duchenne muscular dystrophy: considerations for the design of clinical trials. J Neurol Neurosurg Psychiatry. 2016 Feb;87(2):149-55.

9 Mercuri E, Messina S, Pane M, Bertini E. Current methodological issues in the study of children with inherited neuromuscular disorders. Dev Med Child Neurol. 2008 Jun;50(6): $417-21$.

10 European Medicines Agency. Qualification opinion on stride velocity 95 th centile as a secondary endpoint in Duchenne Muscular Dystrophy measured by a valid and suitable wearable device [Internet]. Amsterdam: European Medicines Agency; 2019 [cited 2020 Jul 29]. Available from: https://www.ema.eu- ropa.eu/en/documents/scientific-guideline/ qualification-opinion-stride-velocity-95thcentile-secondary-endpoint-duchenne-muscular-dystrophy_en.pdf.

11 Alfano LN, Lowes LP, Berry KM, Yin $\mathrm{H}$, Dvorchik I, Flanigan KM, et al. T.P.1: Pilot study evaluating motivation on the performance of timed walking in boys with $\mathrm{Du}$ chenne muscular dystrophy. Neuromuscul Disord. 2014 Oct;24(9-10):860.

12 Bartels B, de Groot JF, Terwee CB. The six-minute walk test in chronic pediatric conditions: a systematic review of measurement properties. Phys Ther. 2013 Apr;93(4):529-41.

13 Banihani R, Smile S, Yoon G, Dupuis A, Mosleh M, Snider A, et al. Cognitive and neurobehavioral profile in boys with Duchenne muscular dystrophy. J Child Neurol. 2015 Oct;30(11):1472-82.

14 Mah JK. Current and emerging treatment strategies for Duchenne muscular dystrophy. Neuropsychiatr Dis Treat. 2016 Jul;12:1795-807.

15 Coravos A, Doerr M, Goldsack J, Manta C, Shervey M, Woods B, et al. Modernizing and designing evaluation frameworks for connected sensor technologies in medicine. NPJ Digit Med. 2020 Mar;3:37.
A Multi-Stakeholder Perspective on a

Novel Digital Endpoint in DMD
Digit Biomark 2021;5:183-190

DOI: $10.1159 / 000517411$ 
16 McDonald CM, Widman LM, Walsh DD, Walsh SA, Abresch RT. Use of step activity monitoring for continuous physical activity assessment in boys with Duchenne muscular dystrophy. Arch Phys Med Rehabil. 2005 Apr; 86(4):802-8.

17 Heberer K, Fowler E, Staudt L, Sienko S, Buckon CE, Bagley A, et al. Hip kinetics during gait are clinically meaningful outcomes in young boys with Duchenne muscular dystrophy. Gait Posture. 2016 Jul;48:159-64.

18 Fowler EG, Staudt LA, Heberer KR, Sienko SE, Buckon CE, Bagley AM, et al. Longitudinal community walking activity in Duchenne muscular systrophy. Muscle Nerve. 2018 Mar; 57(3):401-6.

19 Haberkamp M, Moseley J, Athanasiou D, de Andres-Trelles F, Elferink A, Rosa MM, et al. European regulators' views on a wearable-derived performance measurement of ambulation for Duchenne muscular dystrophy regulatory trials. Neuromuscul Disord. 2019 Jul; 29(7):514-6.

20 Le Moing AG, Seferian AM, Moraux A, Annoussamy M, Dorveaux E, Gasnier E, et al. A movement monitor based on magneto-inertial sensors for non-ambulant patients with Duchenne muscular dystrophy: a pilot study in controlled environment. PLoS One. 2016 Jun;11(6):e0156696.

21 Lilien C, Gasnier E, Gidaro T, Seferian A, Grelet $\mathrm{M}$, Vissière $\mathrm{D}$, et al. Home-based monitor for gait and activity analysis. J Vis Exp. 2019 Aug;150:e59668.

22 Annoussamy M, Seferian AM, Daron A, Péréon Y, Cances C, Vuillerot C, et al. Natural history of Type 2 and 3 spinal muscular atrophy: 2-year NatHis-SMA study. Ann Clin Transl Neurol. 2021 Feb;8(2):359-73.

23 Eliopoulos H, Laforet G, Narayana A, Lucas K, Duda P. Predictors of disease severity and progression in patients with Duchenne muscular dystrophy: a literature review. Eur J Paediatr Neurol. 2017 Jun;21(Suppl 1):e93.
24 Fogel DB. Factors associated with clinical trials that fail and opportunities for improving the likelihood of success: a review. Contemp Clin Trials Commun. 2018 Aug;11:156-64.

25 Duchenne UK. Patient and caregiver perspectives on Duchenne clinical trials survey report [Internet]. Hammersmith: Duchenne UK; 2017 [cited 2020 Aug 16]. Available from: https: //www.duchenneuk.org/news/patientsurvey-report.

26 Powers JH 3rd, Patrick DL, Walton MK, Marquis P, Cano S, Hobart J, et al. Clinician-reported outcome assessments of treatment benefit: report of the ISPOR clinical outcome assessment emerging good practices task force. Value Health. 2017 Jan;20(1):2-14.

27 Servais L, Gidaro T, Seferian A, Gasnier E, Daron A, Ulinici A, et al. P.191Maximal stride velocity detects positive and negative changes over 6- month-time period in ambulant patients with Duchenne muscular dystrophy. Neuromuscul Disord. 2019 Oct;29(Suppl 1):S105.

28 Polhemus AM, Kadhim H, Barnes S, Zebrowski SE, Simmonds A, Masand SN, et al. Accelerating adoption of patient-facing technologies in clinical trials: a pharmaceutical industry perspective on opportunities and challenges. Ther Innov Regul Sci. 2019 Jan;53(1): 8-24.

29 Mercuri E, Muntoni F. Muscular dystrophy: new challenges and review of the current clinical trials. Curr Opin Pediatr. 2013 Dec;25(6): 701-7.

30 Fonseca DA, Amaral I, Pinto AC, Cotrim MD. Orphan drugs: major development challenges at the clinical stage. Drug Discov Today. 2019 Mar;24(3):867-72.

31 US Food and Drug Administration. Clinical outcome assessments (COA) qualification program DDT COA \#000103: ActiMyo [Internet]. Silver Spring: US Food and Drug Administration; 2018 Aug 30 [cited 2020 Jul 28]. Available from: https://www.fda.gov/drugs/ ddt-coa-000103-actimyor.
32 European Medicines Agency, Human Medicines Division. Qualification of novel methodologies for medicine development [Internet]. Amsterdam: European Medicines Agency; 2020 [cited 2020 Jul 29]. Available from: https://www.ema.europa.eu/en/human-regulatory/research-development/scientific-advice-protocol-assistance/qualification-novel-methodologies-medicine-development- 0 .

33 European Medicines Agency, Human Medicines Division. Questions and answers: qualification of digital technology-based methodologies to support approval of medicinal products [Internet]. Amsterdam: European Medicines Agency; 2020 [cited 2021 May 05]. Available from: https://www.ema.europa.eu/ en/documents/other/questions-answersqualification-digital-technology-basedmethodologies-support-approval-medicinal_en.pdf.

34 Foundation for the National Institutes of Health Biomarkers Consortium. Workshop: remote digital monitoring for medical product development [Internet]. Bethesda: Foundation for the National Institutes of Health Biomarkers Consortium; 2020 [cited 2020 Jul 28]. Available from: https://fnih.org/whatwe-do/biomarkers-consortium/programs/ digitalmonitoring.

35 Clay I. Impact of digital technologies on novel endpoint capture in clinical trials. Clin Pharmacol Ther. 2017 Dec;102(6):912-3.

36 Perry B, Herrington W, Goldsack JC, Grandinetti CA, Vasisht KP, Landray MJ, et al. Use of mobile devices to measure outcomes in clinical research, 2010-2016: a systematic literature review. Digit Biomark. 2018 Jan-Apr; 2(1):11-30.

37 Goldsack JC, Dowling AV, Samuelson D, Patrick-Lake B, Clay I. Evaluation, acceptance, and qualification of digital measures: from proof of concept to endpoint. Digit Biomark. 2021 Jan-Apr;5(1):53-64. 